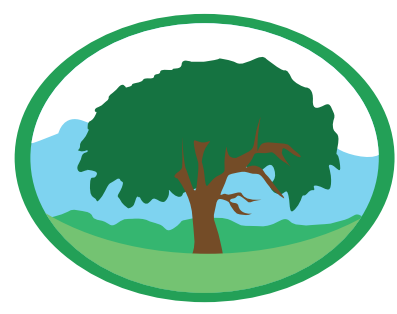

\title{
ANÁLISE DAS PROPRIEDADES FÍSICAS E MECÂNICAS DE GESSO RECICLADO A PARTIR DE RESÍDUOS DA CONSTRUÇÃO CIVIL
}

\author{
VASQUES, C. A. R. ${ }^{1}$; BIRCK, F. B. ${ }^{1}$; ARNOLD, D. M. ${ }^{1}$; KAZMIERCZAK, C. S. ${ }^{2}$. \\ 1 Universidade Feevale/Curso de Engenharia Civil \\ ${ }^{2}$ Universidade do Vale do Rio dos Sinos - UNISINOS/Programa de Pós-Graduação em Engenharia Civil
}

\begin{abstract}
Palavras-chave:
gesso;

resíduos de gesso; reciclagem

de gesso; placas de gesso; gesso acartonado
\end{abstract}

\section{Resumo}

Resíduos de gesso devem ter destinação adequada, pois apresentam potencial de contaminação ambiental. A sua reciclagem é uma das alternativas para o seu reaproveitamento sustentável. Assim, através de uma pesquisa quantitativa, descritiva e experimental, se objetivou analisar as propriedades físicas e mecânicas de gesso reciclado, avaliando a compatibilidade dos produtos obtidos frente as novas especificações técnicas brasileiras para seu uso na construção civil. Resíduos de placas lisas de gesso para forro e de chapas de gesso acartonado (drywall) foram submetidos à moagem e calcinação controladas, e os produtos de reciclagem obtidos foram analisados comparativamente quanto a sua distribuição granulométrica, massa unitária, consistência normal e tempo de pega da pasta, além de dureza, resistência à compressão e à tração na flexão. Os resultados obtidos sugerem que estes resíduos possam ser reciclados à gesso para reutilização na construção civil, porém mais estudos são exigidos para garantir melhor adequação de suas propriedades às normas técnicas brasileiras.

\section{ANALYSIS OF THE PHYSICAL AND MECHANICAL PROPERTIES OF RECYCLED GYPSUM FROM CONSTRUCTION WASTE}

Keywords: gypsum; gypsum plaster waste; gypsum plaster recycling; gypsum plasterboards; drywall sheets

\section{Abstract}

Gypsum plaster waste must have adequate disposal, since they present potential of environmental contamination. Recycling is one of the alternatives for sustainable reuse. Thus, through a quantitative, descriptive and experimental research, the objective was to analyze the physical and mechanical properties of recycled gypsum, evaluating the compatibility of the products obtained against the new Brazilian technical specifications for its use in civil construction. Gypsum waste of plaster board and standard drywall sheets were subjected to controlled grinding and calcination, and the recycled products obtained were analyzed for particle size distribution, unit mass, normal consistency and setting time of the paste, as well as surface hardness, compressive and flexural strength. The results suggest that these residues can be recycled to gypsum for reuse in the construction industry, but more studies are required to ensure better adaptation of their properties to Brazilian standards. 


\section{INTRODUÇ̃̃o}

O gesso é um insumo amplamente usado na construção civil, sendo que seus resíduos contribuem com cerca de $1 \%$ do total gerado neste setor no Brasil (IPEA, 2012). Até 2011 no país, resíduos de gesso não eram passíveis de reaproveitamento segundo a legislação ambiental, sendo considerados como resíduos para os quais não foram desenvolvidas tecnologias ou aplicaçóes economicamente viáveis que permitam a sua reciclagem ou recuperação. A resoluçáo do Conselho Nacional do Meio Ambiente no 431 passou a classificá-los como "resíduos recicláveis para outras destinaçóes" (Classe B), frente ao desenvolvimento de novas tecnologias para o seu reaproveitamento (CONAMA, 2011).

Com a possibilidade de reciclagem, além do ganho sob o aspecto econômico, abre-se a perspectiva de redução do uso dos recursos naturais, diminuindo o impacto ambiental inerente à atividade da extração de gipsita, minério empregado na produção de gesso. Em adição, seu descarte em aterros pode ser mitigado, considerando que a decomposição biológica dos sulfatos constituintes do gesso resulta na produção de sulfeto de hidrogênio, importante contaminante ambiental (LEE et al., 2006). Neste sentido, os resíduos de gesso poderiam ser reciclados e reincorporados a cadeia produtiva da construção civil, porém esta alternativa é praticamente inexistente no Brasil (OLIVEIRA; POLISSENI, 2013).

A reciclagem do gesso pode ser resumida num processo que envolve a calcinação controlada do resíduo, constituído por sulfato de cálcio di-hidratado, à uma forma hemi-hidratada do mesmo sal (BARDELLA; CAMARINI, 2012). Entretanto, vários aspectos tecnológicos são críticos neste processamento, os quais ainda merecem maiores estudos. Neste contexto, o presente estudo objetivou analisar comparativamente as propriedades físicas e mecânicas de gesso reciclado a partir de resíduos de placas lisas para forro e chapas de gesso acartonado (drywall), avaliando a eficácia energética de diferentes condições metodológicas de calcinação, bem como verificando a compatibilidade dos produtos obtidos frente as especificaçôes da nova versão da NBR 13207 (ABNT, 2017a), que estabelece os requisitos para a caracterização, inspeção e recebimento do gesso usado na construção civil para fabricação de elementos, componentes e revestimento de superfícies.

\section{MATERIAL E MÉTODOS}

Foram selecionados Resíduos de Gesso Liso para forro (RGL) e Resíduos de Gesso Drywall do tipo standard (RGD) oriundos de sobras de instalação, os quais não apresentavam qualquer tipo de acabamento superficial. Impurezas aparentes foram retiradas e o revestimento de papel do núcleo de gesso das chapas de drywall foi removido com uma lixadeira de cinta. Os resíduos foram triturados manualmente até uma granulometria igual ou menor que $2 \mathrm{~cm}$ e submetidos à moagem durante 10 minutos em moinho de bolas.

As amostras pulverizadas foram previamente dessecadas a $40^{\circ} \mathrm{C}$ até massa constante. Com $1 \mathrm{~kg}$ de cada resíduo, verificou-se o tempo necessário para desidratação dos resíduos à uma forma reciclada, empregando-se temperaturas de $125^{\circ} \mathrm{C}, 150^{\circ} \mathrm{C}$ e $175^{\circ} \mathrm{C}$. Considerando o teor de pureza próximo a $100 \%$ do sulfato de cálcio di-hidratado constituinte dos resíduos $\left(\mathrm{CaSO}_{4} \cdot 2 \mathrm{H}_{2} \mathrm{O}\right)$, o tempo de calcinação foi definido pelo tempo necessário para que ocorresse uma redução da massa molar equivalente à perda de $75 \%$ de água de cristalização, ou seja, retirada suficiente de água para sua conversão total à forma hemi-hidratada $\left(\mathrm{CaSO}_{4} \cdot 1 / 2 \mathrm{H}_{2} \mathrm{O}\right)$. Assim, considerando a massa molar do di-hidratado de 172 g e do hemi-hidratado de 145 $\mathrm{g}$, as amostras ficaram em calcinação durante o tempo necessário para que ocorresse uma redução de 15,7\% da sua massa. Para esta determinação, a amostra foi pesada a cada 60 minutos durante a calcinação, até que se alcance esta redução em massa com variação de \pm $1 \%$.

Após determinação dos tempos de calcinação em função da temperatura, definiu-se as condiçôes de $150^{\circ} \mathrm{C}$ por 2 horas para obtenção das amostras cujas propriedades físicas e mecânicas foram ensaiadas. Estas condições foram selecionadas a partir do critério de menor consumo energético envolvido no processamento. Para esta análise, mediu-se com um amperímetro o valor médio da corrente instantânea " $P$ " (em Amperes) de alimentação da estufa após 
estabilização das diferentes temperaturas testadas, além de se determinar a fração de tempo em que a resistência se mantinha em funcionamento durante o período do ciclo on-off do equipamento (estufa termostatizada). Esta fração foi calculada pela relação entre o tempo ligado da resistência $\left(\mathrm{t}_{\mathrm{on}}\right)$ e o tempo total do ciclo de operação on-off $\left(\mathrm{t}_{\text {on-off }}\right)$. Considerando a tensão nominal da rede de $220 \mathrm{~V}$, determinou-se a potência de operação da estufa através do produto entre corrente e tensão. $\mathrm{O}$ consumo energético (CE) para cada temperatura foi estimado em $\mathrm{kW} . \mathrm{h}$, usando o produto entre a potência e o tempo total de calcinação da amostra $t_{\text {cal }}$ (em horas), conforme Equação 1.

$$
\mathrm{CE}=\left\{\left[\mathrm{I} \cdot\left(\mathrm{t}_{\text {on }} / \mathrm{t}_{\text {ton-off }}\right) \cdot 220\right] / 1000\right\} \cdot \mathrm{t}_{\text {cal }}
$$

Além dos resíduos calcinados $\left(\mathrm{RGL}_{\text {cal }}\right.$ e $\left.\mathrm{RGD}_{\text {cal }}\right)$, as análises físicas e mecânicas também foram realizadas em amostras de Gesso Comercial para fundição (GC), usado como referência para as análises. A massa unitária das amostras foi verificada conforme descrito pela NBR 12127 (ABNT, 2017b), que especifica o método para determinação das propriedades físicas do gesso na forma de pó. A partir da adaptação do método descrito por esta mesma norma, a determinação da granulometria das amostras foi realizada em peneiras com aberturas de malha de 0,425 mm, 0,300 $\mathrm{mm}, 0,250 \mathrm{~mm}, 0,150 \mathrm{~mm}$ e $0,075 \mathrm{~mm}$. Através da obtenção da melhor equação que descreve o comportamento da distribuição granulométrica, foram estimados os diâmetros de grão para $10 \%\left(\mathrm{~d}_{10}\right)$ e $60 \%$ $\left(\mathrm{d}_{60}\right)$ de material passante, usados para determinação do coeficiente de náo uniformidade $\left(\mathrm{C}_{\mathrm{nu}}\right)$, obtido pela relação d60/d10 (CAPUTO, 1996).

Consistência normal e tempo de pega foram ensaiados usando-se um aparelho de Vicat modificado e convencional, respectivamente, seguindo procedimentos descritos pela NBR 12128 (ABNT, 2017c), que especifica o método para determinação das propriedades físicas da pasta de gesso. $\mathrm{O}$ resultado do ensaio de consistência normal foi usado para determinação da relação água/gesso empregado para preparação das amostras de pasta de gesso submetidas à análise de tempo de início e fim de pega, bem como para confecção dos corpos de provas usados para análise do desempenho mecânico do material.
Os ensaios de dureza e resistências à compressão foram realizados sobre corpos de prova cúbicos $(50$ $\mathrm{mm}$ de aresta), seguindo procedimentos prescritos na NBR 12129 (ABNT, 2017d), que especifica o método para determinação das propriedades mecânicas gesso. A tração na flexão foi ensaiada com corpos de provas prismáticos (40 x 40 x $160 \mathrm{~mm}$ ), conforme procedimento adaptado a partir da NBR 12775 (ABNT, 2018), que estabelece o método para determinação das propriedades mecânicas de placas lisas de gesso para forro autoportante. Todos os ensaios para análise do desempenho mecânico foram realizados em corpos de prova de 7 e 28 dias de idade.

Os resultados foram apresentados como médias \pm desvio padrão, sendo as médias comparadas através do teste $\mathrm{t}$ de Student para amostras independentes ou pareadas, empregando-se uma significância estatística para $\mathrm{p} \leq 0,01$. Através de modelos de regressão linear foram determinadas as equaçôes das linhas de tendência (equação de regressão) usadas para descrever o comportamento das curvas de distribuição granulométrica.

\section{RESULTADOS E DISCUSSÃO}

\section{Calcinação}

Todas as temperaturas de calcinação testadas $\left(125^{\circ}\right.$ $\mathrm{C}, 150^{\circ} \mathrm{C}$ e $175^{\circ} \mathrm{C}$ ) foram capazes de promover a redução prevista de massa das amostras para conversão dos resíduos de gesso à uma forma hemi-hidratada. Como apresentado na Tabela 1, a calcinação realizada a $150^{\circ} \mathrm{C}$ foi energeticamente mais eficiente que as demais para obtenção da desidratação desejada das amostras, sendo que a mesma operaçáo executada a $125^{\circ}$ e $175^{\circ} \mathrm{C}$ demandaram $18,4 \%$ e $43,2 \%$ a mais de consumo elétrico, respectivamente. 
Tabela 1. Tempo de calcinação dos resíduos de gesso em função das diferentes temperaturas avaliadas

\begin{tabular}{|c|c|c|c|c|}
\hline $\begin{array}{l}\text { Temperatura } \\
\text { de } \\
\text { calcinação } \\
\left({ }^{\circ} \mathrm{C}\right)\end{array}$ & Amostra & $\begin{array}{l}\% \text { de } \\
\text { perda } \\
\text { de } \\
\text { massa }\end{array}$ & $\begin{array}{l}\text { Tempo de } \\
\text { calcinação } \\
\text { (h) }\end{array}$ & $\begin{array}{l}\text { Gasto } \\
\text { energético } \\
(\mathrm{kW} \cdot \mathrm{h})^{*}\end{array}$ \\
\hline \multirow[t]{2}{*}{125} & RGL & 15,5 & 4 & 1,48 \\
\hline & RGD & 15,1 & & \\
\hline \multirow[t]{2}{*}{150} & RGL & 15,7 & 2 & 1,25 \\
\hline & RGD & 15,5 & & \\
\hline \multirow[t]{2}{*}{175} & RGL & 15,8 & 2 & 1,79 \\
\hline & RGD & 15,8 & & \\
\hline
\end{tabular}

*Valores referentes ao consumo energético para calcinação de $1 \mathrm{~kg}$ de amostra.

No estudo de Pinheiro (2011), o consumo energético para calcinação de resíduos de gesso à $150^{\circ} \mathrm{C}$ por 1 hora foi de $0,520 \mathrm{~kW} . \mathrm{h} / \mathrm{kg}$, valor similar ao encontrado em condições laboratoriais no presente estudo $(0,625 \mathrm{~kW} \cdot \mathrm{h} / \mathrm{kg})$. Entretanto, apesar de resultados da literatura indicarem que 1 hora de calcinação à temperatura constante de $150^{\circ} \mathrm{C}$ é o suficiente para obtençáo de um reciclado com altos teores de hemi-hidratado (PINHEIRO; CAMARINI, 2015; GERALDO et al., 2017), nesta condição foi verificado uma redução de massa de aproximadamente $10 \%$ para ambas as amostras, RGL e RGD, equivalendo a cerca de $65 \%$ de conversão da forma di-hidratada à hemi-hidratada.

A Figura 1 apresenta a redução de massa durante as 4 horas de calcinação a $125^{\circ} \mathrm{C}$, demonstrando uma cinética de desidratação muito similar entre ambas amostras. Pode-se observar que a taxa de desidratação se manteve relativamente constante durante a primeira metade deste período, reduzindo à medida que se aproximou dos 15\% de perda de massa, o que possivelmente equivale a conversão quase total $\mathrm{da}$ forma di-hidratado à hemi-hidratada. Neste sentido, Ferreira et al. (2003) verificaram através do balanço de massa que só em temperaturas maiores, entre $195^{\circ}$ e $240^{\circ} \mathrm{C}$, a cinética de desidrataçấo do gesso evolui para a conversão do hemi-hidratado à forma anidra de sulfato de cálcio.

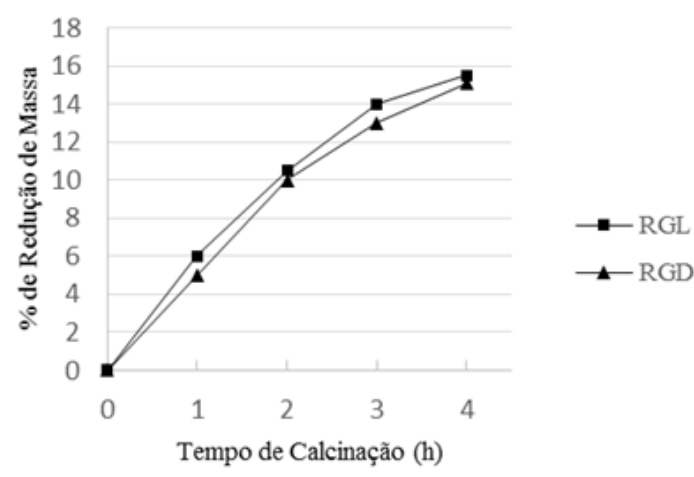

Figura 1. Desidratação dos resíduos de gesso ao longo de 4 horas de calcinação à $125^{\circ} \mathrm{C}$

\section{Análise do pó de gesso reciclado}

A nova versão da NBR 13207 (ABNT, 2017a) especifica que no mínimo $90 \%$ da amostra homogeneizada de pó de gesso deve apresentar grãos com diâmetro menor que $0,290 \mathrm{~mm}$, o que foi observado para todas as amostras deste estudo. Caso mais de $90 \%$ dos grãos possua diâmetro menor que $0,210 \mathrm{~mm}$, esta norma classifica o material como gesso para revestimento, do contrário será considerado gesso para fundição. Assim, considerando que o percentual passante pela peneira de abertura $0,150 \mathrm{~mm}$ ficou acima dos 90\%, tanto para o gesso comercial como para os resíduos reciclados, as amostras seriam classificadas como gesso para revestimento segundo a nova versão desta norma. Apesar disto, a amostra GC foi comercialmente obtida rotulada como "gesso fino para fundição", bem como os resíduos usados não eram provenientes de gesso para revestimento. De fato, a versão anterior da NBR 13207, vigente até junho de 2017, estabelecia somente o tempo de pega como critério de classificação quanto ao uso (revestimento ou fundição), sendo a distribuição granulométrica empregada apenas para classificar o gesso como "fino ou grosso". Entretanto, esta última classificação não consta mais na versão da norma em vigor.

Como visualizado na Figura 2, o perfil de distribuição granulométrica de RGL e RGD foi similar, tanto antes como após a calcinação. O gesso comercial apresentou grãos significativamente mais finos que as demais amostras, com 9,0\% de material retido acumulado na peneira abertura $0,075 \mathrm{~mm}$, contra $58,3 \%$ e $48,5 \%$ de $\mathrm{RGL}_{\text {cal }}$, $\mathrm{RGD}_{\text {cal }}$, respectivamente. As curvas granulométricas dos resíduos apresentaram 
um evidente deslocamento para a esquerda após calcinação, se aproximando do perfil verificado para o gesso comercial (Figura 2) e sugerindo que a conversão da forma di-hidratada para a hemi-hidratada foi acompanhada por uma redução do diâmetro dos grãos.

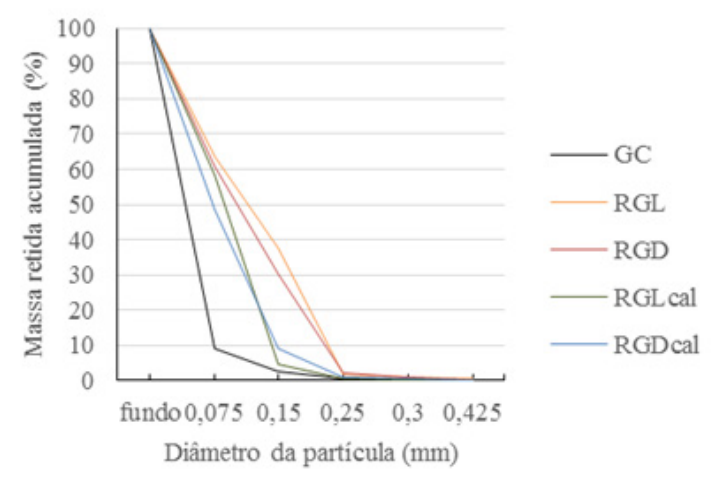

Figura 2. Curvas de distribuição granulométrica

Na mesma direção, Rossetto et al. (2016) testaram tempos crescentes de calcinação (1 a 6 horas) à temperatura constante de $150^{\circ} \mathrm{C}$, verificando uma redução significativa dos diâmetros dos grãos nas primeiras 3 horas de desidratação. Assim, alterações da morfologia dos cristais de sulfato de cálcio em função de seu grau de hidratação poderiam ser uma provável causa para esta variação granulométrica.

Considerando a distribuição granulométrica a partir do percentual passante em função do diâmetro do grão (Figura 3), foram obtidas equações através de um modelo logarítmico que melhor descreveu os perfis observados, apresentando maiores coeficientes de determinação $\left(\mathrm{R}^{2}\right)$ que outros modelos estatísticos de regressão linear testados.
A partir destas equaçóes foram estimados os diâmetros de grão para 10\% e 60\% de material passante, usados para determinaçáo do coeficiente de não uniformidade (Cnu). Este coeficiente indica a uniformidade do diâmetro dos grãos, sendo que valores de Cnu menores que 5 apontam uma distribuição "muito uniforme", e maiores que 15 "não uniforme" (CAPUTO, 1996). Os resultados apontaram a não uniformidade da distribuição do gesso comercial ( $\mathrm{Cnu}>150)$, enquanto os reciclados de RGL (Cnu = $4,60)$ e RGD (Cnu $=5,96)$ apresentaram uma maior uniformidade dos grãos.

O gesso comercial, cuja massa unitária foi de $636,0 \pm 4,2 \mathrm{~kg} / \mathrm{m} 3$, foi a única amostra que atingiu as especificaçốes da NBR 13207 (ABNT, 2017a), que preconiza valores iguais ou maiores que $600 \mathrm{~kg} / \mathrm{m} 3$. As amostras calcinadas de RGL e RGD, apresentaram massa unitária de $465,7 \pm 10,0 \mathrm{~kg} / \mathrm{m} 3$ e $500,0 \pm 3,5$ $\mathrm{kg} / \mathrm{m} 3$, respectivamente, significativamente menor que do gesso comercial, reproduzindo resultados similares aos encontrados na literatura (CAMARINI; LIMA; PINHEIRO, 2016). Estes resultados podem estar relacionados com a maior uniformidade da distribuição granulométrica das amostras de resíduos reciclados frente ao gesso comercial, gerando maior quantidade de espaços vazios, o que foi sugerido através da análise do coeficiente de náo uniformidade.

\section{Análise da pasta de gesso reciclado}

A consistência normal foi obtida a partir da relação água/gesso necessária para formar uma pasta onde a sonda cônica do aparelho de Vicat modificado foi
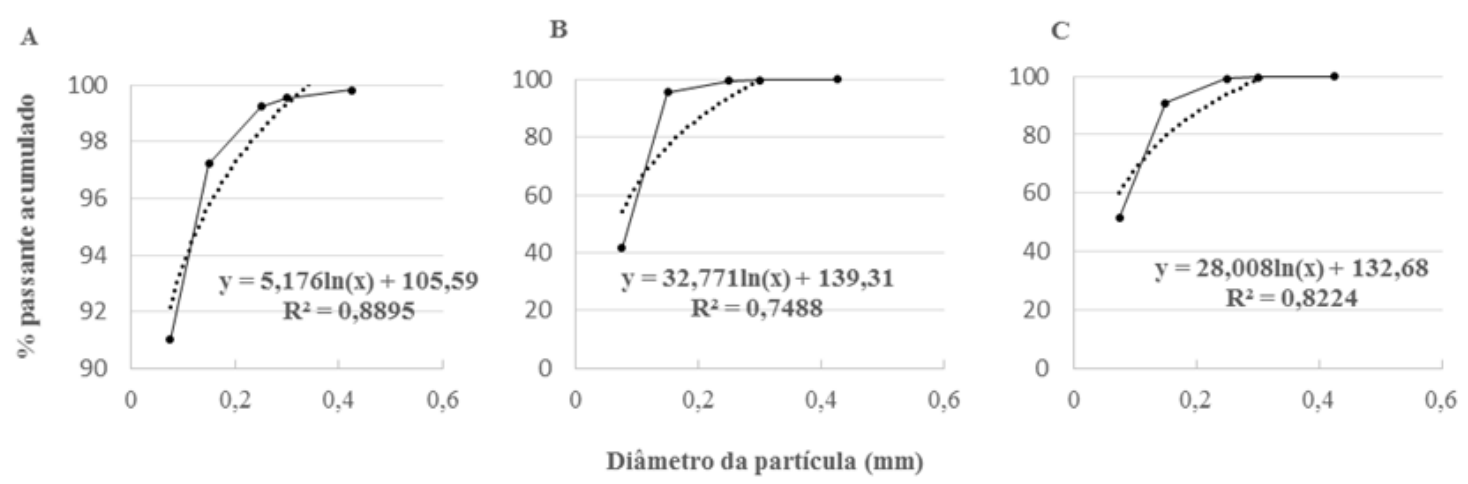

Figura 3. Curvas de distribuição granulométrica com suas respectivas equações de regressão. *Curvas de distribuição granulométricas para GC (3A) e reciclados de RGL (3B) e RGD (3C) 
capaz de penetrá-la $30 \pm 2 \mathrm{~mm}$, segundo condiçóes estabelecidas na NBR 12128 (ABNT, 2017c). Para as amostras calcinadas de RGL e RGD, a consistência normal foi verificada com uma relação água/gesso igual a 0,8 e 0,9, respectivamente. Para obtenção de uma consistência normal para GC foi necessária uma relação de 0,55 , cerca de $30 \%$ menor que a observada para os reciclados. Ao contrário do sugerido por Nita, Pileggi e Cincotto (2004), o aumento da relação água/ gesso para o reciclado, em comparação ao comercial, não se deu em função de uma maior área superficial para adsorção de água e reaçóes de hidratação deste material, uma vez que os grãos das amostras calcinadas apresentaram diâmetros maiores que o GC, como evidenciado ao comparar as curvas de distribuição granulométrica (Figura 2).

Usando uma relação água/gesso igual a 1, Camarini et al. (2016) obtiveram pastas de gesso reciclado com baixa trabalhabilidade, observando uma imersão da sonda do aparelho de Vicat modificado de apenas 5 $\mathrm{mm}$ sobre a superfície da amostra, muito abaixo dos $30 \mathrm{~mm}$ preconizado pela NBR 12128 para obtenção de uma consistência normal (ABNT, 2017c). Estes resultados divergem dos encontrados no presente estudo, onde foi possível obter uma trabalhabilidade adequada mesmo com uso de menores quantidades de água, o que provavelmente se relaciona as diferenças dos perfis granulométricos dos materiais usados, uma vez que Camarini et al. (2016) obtiveram um reciclado com grãos maiores (cerca de $90 \%>0,074 \mathrm{~mm}$ ).

Empregando os traços definidos a partir do ensaio de consistência normal, foram preparadas pastas de gesso com as amostras de resíduos calcinados e com o gesso comercial, as quais foram submetidas ao ensaio de tempo de pega (Tabela 2). A velocidade de hidratação de RGDcal foi evidentemente maior que as observadas para as demais amostras, tendo precoce início e fim de pega. Porém, o perfil de tempo de pega de todas as amostras se aproximou ao estabelecido para o gesso para fundição, segundo critérios definidos pela NBR 13207 (ABNT, 2017a). Esta norma estabelece para este tipo de uso um tempo de início de pega menor ou igual a 10 minutos, e menor ou igual a 20 minutos para seu fim. De acordo com esta norma, o gesso para uso como revestimento necessita um tempo maior de hidratação antes do seu endurecimento (tempo final de pega maior que 35 minutos).

Tabela 2. Tempos de início e fim de pega.

\begin{tabular}{cccc}
\hline \multirow{2}{*}{ Amostra } & \multicolumn{2}{c}{ Tempo de pega $(\min )$} & \multirow{2}{*}{$\begin{array}{c}\text { Variação } \\
(\mathrm{min})\end{array}$} \\
\cline { 2 - 3 } & Inicio & Fim & (13 \\
\hline GC & 10 & 23 & 7 \\
\hline RGL $_{\mathrm{cal}}$ & 12 & 19 & 4 \\
\hline RGD $_{\mathrm{cal}}$ & 7 & 11 & 4 \\
\hline
\end{tabular}

\section{Análise do gesso reciclado no estado endurecido}

A dureza superficial verificada para as amostras de gesso reciclado foi significativamente menor que a do gesso comercial. Como ilustrado na Figura 4A, apenas GC alcançou o limite mínimo de dureza de $20 \mathrm{MPa}$ estabelecido pela NBR 13207 (2017a), sendo que RGL com 28 dias foi a amostra do material reciclado que atingiu valores mais próximos a este limite $(18,2 \pm 2,8$ $\mathrm{MPa})$. As amostras de gesso reciclado apresentaram um menor desempenho de resistência à compressão e tração na flexão comparado ao gesso comercial (Figuras 4B e 4C). A versão anterior da NBR 13207 especificava um valor mínimo de $8,4 \mathrm{MPa}$ de resistência à compressão para uso de gesso na construçáo civil, porém a sua versão atual não exige mais limites mínimos para esta propriedade mecânica. Além do gesso comercial, apenas a amostra RGL com 28 dias atingiu este limite, apresentando uma média de 8,7 $\pm 0,6 \mathrm{MPa}$ para resistência à compressão axial. Para todas propriedades mecânicas testadas foi demonstrado um aumento significativo do desempenho do material reciclado com 28 dias, em relação aos 7 dias de idade, o que também foi observado em outros estudos (ERBS et al., 2015; GERALDO et al., 2017). Assim, é provável que a resistência definitiva do gesso reciclado seja alcançada somente tardiamente, com quatro ou mais semanas, dependendo das condiçóes ambientais de secagem. 

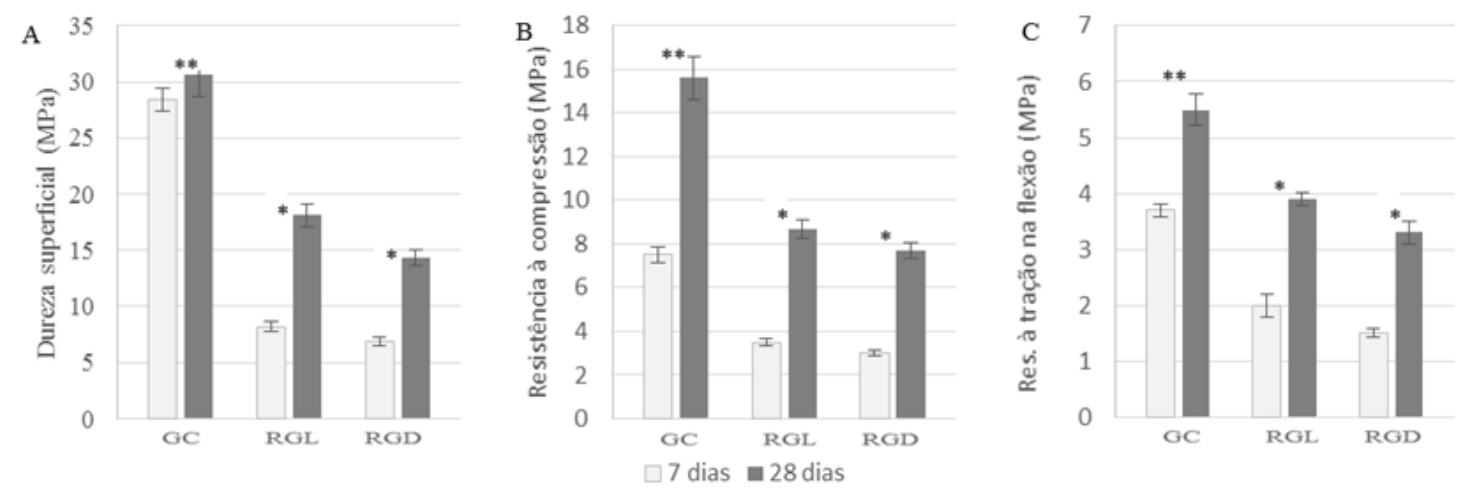

Figura 4. Propriedades mecânicas das amostras analisadas

* $p<0,01: 7$ dias versus 28 dias (teste t para amostras pareadas, $n=6$ )

** $p<0,01: G C$ versus RGL e RGD (teste t para amostras independentes, $n=6$ )

\section{CONCLUSÃO}

A desidratação dos resíduos de gesso para produçáo de uma forma hemi-hidratada parece ocorrer com as temperaturas propostas neste estudo, sendo a calcinação à $150^{\circ} \mathrm{C}$ por 2 horas, a condição metodológica com maior eficiência energética entre as avaliadas. A distribuição granulométrica dos reciclados de resíduos de gesso liso e acartonado apresentaram perfis similares, com grãos muito uniformes, o que pode explicar a baixa massa unitária em relação ao gesso comercial. Foi possível determinar um traço com consistência normal, apresentando boa fluidez e trabalhabilidade sem uso de aditivos, além de definir um tempo de pega que se aproximou ao do gesso comercial para fundição.

$\mathrm{O}$ desempenho mecânico relacionado à dureza e resistências a compressão e flexão do gesso reciclado foi menor que o gesso comercial. A maior relação água/gesso necessária para confecção de pastas com consistência normal do gesso reciclado, em comparação ao comercial, provavelmente interferiu no seu desempenho mecânico.

Neste sentido, é possível que um processo de moagem mais eficiente, reduzindo o tamanho dos grãos e levando a uma melhor graduaçáo, permita um aumento da massa unitária e a redução da relação água/ gesso reciclado, mantendo sua consistência normal com níveis de desempenho mecânico similares ao do gesso comercial. Assim, os resultados obtidos sugerem que tanto os resíduos de gesso liso como acartonado possam ser usados como fonte de reciclagem para sua reutilização na construção civil, porém mais estudos são exigidos para que se garanta melhor adequação de suas propriedades às exigências das normas técnicas vigentes.

\section{LITERATURA CITADA}

ABNT: Associação Brasileira de Normas Técnicas. NBR 13207: Gesso para construção civil - requisitos. Rio de Janeiro, 2017a.

NBR 12127: Gesso para construção - determinação das propriedades físicas do pó. Rio de Janeiro, 2017b.

NBR 12128: Gesso para construção - determinação das propriedades físicas da pasta. Rio de Janeiro, 2017c.

NBR 12129: Gesso para construção - determinação das propriedades mecânicas. Rio de Janeiro, 2017d.

NBR 12775: Placas lisas de gesso para forro autoportante - método de ensaio. Rio de Janeiro, 2018.

BARDELLA, P.S., CAMARINI, G. Recycled Plaster: Physical and Mechanical Properties. Advanced Materials Research, v.374-377, p.1307-1310, 2012.

CAMARINI, G.; LIMA, K.D.S.; PINHEIRO, S.M.M. Investigation on gypsum plaster waste recycling: an eco-friendly material. Green Materials, v.3, n.4, p.104-112, 2016.

CAMARINI, G.; PINHEIRO, S.M.M. Microstructure of recycled gypsum plaster by SEM. Advanced Materials Research, v.912-914, p.243-246, 2014.

CAMARINI, G.; PINTO, M.C.C.; MOURA, A.G.; MANZO, N.R. Effect of citric acid on properties of recycled gypsum plaster to building components. Construction and Building Materials, v.124, p.383-390, 2016.

CAPUTO, H.P. Mecânica dos solos e suas aplicaçôes. Rio de Janeiro: LTC, 1996. 234p.

CONAMA: Conselho Nacional do Meio Ambiente. Resolução no 431. Brasília: Diário Oficial da União no 99, 2011. p.123. 
ERBS, A.; NAGALLI, A.; MYMRINE, V.; CARVALHO, K. Q. Determinação das propriedades físicas e mecânicas do gesso reciclado proveniente de chapas de gesso acartonado. Cerâmica, v.61, p.482-487, 2015.

FERREIRA, R.A.S.; SILVA, I.A.; YADAVA, Y.P.; BARAÚNA, O.S.; AGUIAR, J.A.O. Estudo da cinética de decomposição dos diferentes tipos de gipsita do Araripe pernambucano. In: Annual Meeting of the Brazilian Ceramic Society, 47, 2003, João Pessoa. Proceedings ... São Paulo: ABCERAM, 2003. p.2130-2140.

GERALDO, H.R.; PINHEIRO, S.M.M.; SILVA, J. S.; ANDRADE, H.M.C.; DWECK, J.; GONÇALVES, J.P.; CAMARINI, G. Gypsum plaster waste recycling: A potential environmental and industrial solution. Journal of Cleaner Production, v.164, p.288-300, 2017.

IPEA: Instituto de Pesquisa Econômica Aplicada. Diagnóstico dos resíduos sólidos da construção civil - relatório de pesquisa. Brasília: Livraria IPEA, 2012. 34p.

LEE, S.; XU, Q.; BOOTH, M.; TOWNSEND, T.G.; CHADIK, P.; BITTON, G. Reduced sulfur compounds in gas from construction and demolition debris landfills. Waste Management, v.26, n.5, p.526-533, 2006.

NITA, C.; PILEGGI, R.G.; CINCOTTO, M.A.; JOHN, V.M. Estudo da reciclagem do gesso de construção. In: Conferência Latino-americana de Construção Sustentável, 1, e Encontro Nacional de Tecnologia do Meio-ambiente, 10, 2004, São Paulo. Anais ... São Paulo: CLACS; ENTAC, 2004. CD Rom.

OLIVEIRA, T.M.; POLISSENI, A.E. Reciclagem de gesso: potencial de aplicaçáo. REUCP, v.8, n.1, p.40-45, 2013.

PINHEIRO, S.M.M. Gesso reciclado: avaliação das propriedades para uso em componentes. 2011. 304f. Tese (Doutorado em Engenharia Civil) - Faculdade de Engenharia Civil, UNICAMP, Campinas.

PINHEIRO, S.M.M.; CAMARINI, G. Characteristics of Gypsum Recycling in Different Cycles. International Journal of Engineering and Technology, v.7, n.3, p.215-218, 2015.

ROSSETTO, J.R.M.; CORREIA, L.S.; GERALDO, R.H.; CAMARINI, G. Gypsum plaster waste recycling: analysis of calcination time. Key Engineering Materials, v.668, p.312-321, 2016. 